\title{
Effect of Long-Term Repeated Interval Rehabilitation on the Gross Motor Function Measure in Children with Cerebral Palsy
}

\author{
Christina Stark ${ }^{1,2,3, *(1)} \quad$ Ibrahim Duran ${ }^{3, *(1)}$ Kyriakos Martakis ${ }^{1,4,5}$ Karoline Spiess ${ }^{3}$ \\ Oliver Semler ${ }^{1,2}$ Eckhard Schoenau ${ }^{1,2,3}$
}
${ }^{1}$ Department of Pediatrics, University of Cologne, Medical Faculty and University Hospital, Cologne, Germany
${ }^{2}$ Cologne Centre for Musculoskeletal Biomechanics (CCMB), University of Cologne, Medical Faculty and University Hospital, Cologne, Germany
${ }^{3}$ Center of Prevention and Rehabilitation, University of Cologne, Medical Faculty and University Hospital, Cologne, Germany
4 Department of International Health, Maastricht University, School CAPHRI, Care and Public Health Research Institute, Maastricht, The Netherlands
${ }^{5}$ Department of Pediatric Neurology, University Children's Hospital (UKGM) and Medical Faculty, Justus Liebig University of Giessen, Giessen, Germany

Neuropediatrics 2020;51:407-416.

\begin{abstract}
Address for correspondence Christina Stark, MSc, Department of Pediatrics, University of Cologne, Medical Faculty and University Hospital, Kerpener Street 62, 50937 Cologne, Germany (e-mail: christina.stark@uk-koeln.de).
\end{abstract}

\begin{abstract}
Keywords

- cerebral palsy

- gross motor function

- GMFM-66

- effect size

- long-term

Background The efficacy of interventions for cerebral palsy (CP) has been frequently investigated with inconclusive results and motor function measured by the Gross Motor Function Measure (GMFM-66) is common.

Objective In this observational analysis, we quantify the GMFM-66 change scores of the second and third year of a multimodal rehabilitation program (interval rehabilitation including home-based, vibration-assisted training) in children with CP.

Methods The study was a retrospective analysis of children with CP (2-13 years) participating for a second $(n=262)$ and third year $(n=86)$ in the rehabilitation program with GMFM-66 scores at start (M0), after 4 months (M4) of intensive training, and after 8 months of follow-up (M12). A method was previously developed to differentiate between possible treatment effects and expected development under standard of care for GMFM-66 scores using Cohen's d effect size (ES; size of difference). Results After the treatment phase of 4 months (M4) in the second year, 125 of 262 children were responder $(E S \geq 0.2)$ and 137 children nonresponder $(E S<0.2)$; mean $E S$ for nonresponder was -0.212 (trivial) and for responder 0.836 (large). After M4 in the third year, 43 children of 86 were responder ( $E S=0.881$ [large]) and 43 nonresponder (ES $=-0.124$ [trivial]).

Discussion and Conclusion Repeated rehabilitation shows a large additional treatment effect to standard of care in $50 \%$ of children which is likely due to the intervention, because in the follow-up period (standard of care), no additional treatment effect was observed and the children followed their expected development.
\end{abstract}

Shared first authors.

received

April 2, 2020

accepted

July 2, 2020

published online

October 16, 2020
(C) 2020 Georg Thieme Verlag KG

Stuttgart - New York
DOI https://doi.org/

10.1055/s-0040-1715489.

ISSN 0174-304X. 


\section{Introduction}

Cerebral palsy (CP) is defined as a group of permanent, but not unchanging, disorders of movement and/or posture due to a nonprogressive lesion in the developing brain ${ }^{1}$ and results in muscle weakness, secondary consequences (like muscle loss, contractures, and bone deformities), and dependence on external support in daily life. With a prevalence of 2 to 3 per 1,000 children born alive, ${ }^{1} \mathrm{CP}$ represents the largest group of physical disabilities in early childhood with high socioeconomic impact for the health care system. Lifetime costs in the United States in 2003 were in total $\$ 11.5$ billion ( $€ 10.4$ billion) for persons with CP born in 2000 of which direct medical costs (e.g., assistive devices, therapies, rehabilitation, and long-term care) were estimated with $\$ 1.175$ billion ( $€ 1.04$ billion; $10.2 \%)^{2}$

In the last decade, the efficacy of interventions for $\mathrm{CP}$ has been frequently investigated. Motor function is the most common outcome parameter. Common interventions include orthopaedic and neurosurgical procedures, antispasticity pharmacotherapy, and motor learning programs. ${ }^{3}$ Nonpharmacological, nonoperative treatment options (as motor learning programs) require moderate-to-intense exercise intensities, like resistance, locomotor, or postural training. ${ }^{4}$ These conservative approaches are investigated in the field of $\mathrm{CP}$, but no conclusive statements can be made on effectiveness up to date. ${ }^{4}$ The identification of responders and nonresponders is also becoming increasingly important for quality management in rehabilitation. In addition, the identification of useful therapies and the avoidance of harmful therapies are important for patients and their families.

Normally, research findings are based on standardized clinical trials. However, long-term results on treatment outcomes for $\mathrm{CP}$ and the availability of clinical routine evidence are missing. But particularly, the clinician and children and families are interested in long-term results of possible interventions and therefore they should be investigated. ${ }^{5}$

The Gross Motor Function Measure (GMFM-66) is the most commonly used measure for gross motor function in children and adolescents with $\mathrm{CP}^{6,7}$ An increase is normally interpreted as a response to intervention. However, an improvement under standard of care can be expected, especially in young children. ${ }^{6,8}$ Very often mean differences are reported not taking natural progression into account. Until recently, it was unclear how much of an increase of the GMFM-66 was due to intervention or expected development under standard of care. Several attempts to overcome these problems have been described previously. ${ }^{9}$

We developed a method, which allows the quantification of the progress of GMFM- 66 scores to differentiate between possible treatment effects and expected development under standard of care. ${ }^{9}$ We also analyzed the effect of a standardof-care (Germany) interval rehabilitation program combined with home-based, vibration-assisted training on motor function in children with CP, "Auf die Beine (on your feet)," and were able to show a large effect size (ES) for the GMFM- $66 .{ }^{9}$

The rehabilitation program "Auf die Beine" has been previously described. ${ }^{10-14}$ It can be repeated after initial participation. In this observational analysis, we will quantify changes of GMFM-66 scores of a long-term ("Auf die Beine" repeated consecutively for a second and third years) repeated interval rehabilitation program with home-based, vibrationassisted training on motor function in children with $\mathrm{CP}$.

\section{Methods}

The present study was a single-center retrospective analysis of prospectively collected data of children with $\mathrm{CP}$ who participated in the rehabilitation program "Auf die Beine" at the Centre of Prevention and Rehabilitation (University of Cologne, Germany) from January 2006 to December 2018. After receiving written informed consent from the legal representative of the child, clinical data were stored in a prospective single-center patient registry. The Ethics Committee of the University of Cologne approved this registry (16-269). A detailed description of the registry can be found at: www.germanctr.de (DRKS0001131).

The inclusion criteria included the diagnosis of $\mathrm{CP}$, age between 2 and 13 years, and valid GMFM-66 examinations at start (M0, month zero), after intervention (month 6 [M6] for the first year and month 4 [M4] for the second and third years), and M12 (1 year after M0; - Fig. 1). More information about the measurement of the GMFM-66 is explained at "assessment of motor function." Exclusion criteria were no diagnosis of $\mathrm{CP}$, age exceeding the defined age range for inclusion, other genetic syndromes or severe chronic diseases, and incomplete datasets. A total of 729 patients participated in the first year (complete GMFM-66 measurements for M0, M6, and M12). Of them, 262 patients (30\%) received a rehabilitation treatment for a second year (complete GMFM-66 measurements for M0, M4, and M12) and 86 patients $(10 \%$ of initial cohort and $33 \%$ of participants of second year) received treatment for a third year (complete GMFM-66 measurements for M0, M4, and M12).

\section{Rehabilitation Protocol}

The rehabilitation program "Auf die Beine" has been previously described ${ }^{10-14}$ and is part of the health care available in Germany. "Auf die Beine" combines intensive, goal-directed training during inpatient stays combined with whole body vibration (WBV) as a home training program for 6 months (-Fig. 1). For the WBV training, a side-alternating platform (System Galileo, Novotec Medical, Pforzheim, Germany) is used and provided by the center. Side-alternating vibration stimuli provoke spinal reflexes and muscle contractions inducing involuntary muscle stimulation. ${ }^{15-18}$

During inpatient stays, participants receive 4 to 5 hours of physiotherapy daily (goal directed, high intensity, and massed practice), involving training apparatus if applicable and three WBV sessions per day. Children, parents, and therapists work following the child's individual goals, respecting the framework of the World Health Organization (WHO) International Classification of Functioning, Disability, and Health (ICF), twice a day, in a functional physiotherapy setting for 1 hour. Additionally, three components are added: functional resistance training, pool therapy, and treadmill 


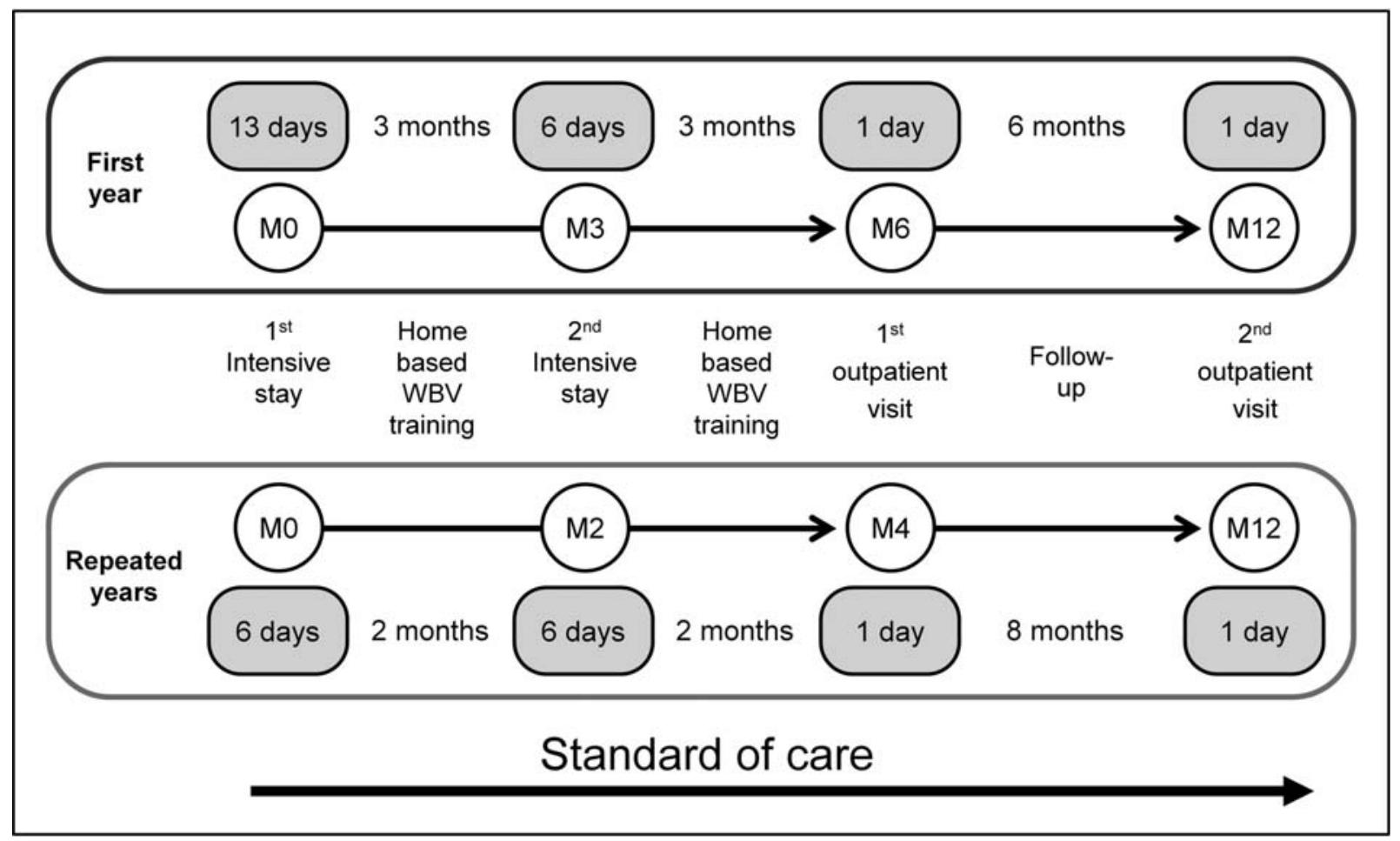

Fig. 1 Rehabilitation program Auf die Beine (on your feet).

training with or without body weight support. Each of the additional components is applied two to three times weekly.

During the first inpatient stay, the children and parents are familiarized with the WBV home-training protocol. Each WBV session is $3 \times 3$ minutes long. The training protocol includes standardized exercises according to the patient's individual goals and is applied ten times per week for $3 \times 3$ minutes. Exercises on the platform include standing (if possible with dynamic squatting), sitting and four-point position. Compliance is facilitated and monitored by a training log.

The vibration frequency is adjusted according to the individual goals and is chosen according to current recommendations: 5 to $12 \mathrm{~Hz}$ for balance and proprioceptive training, 12 to $20 \mathrm{~Hz}$ for muscle function improvement (repetitive contraction and muscle relaxation enabled), and 20 to $27 \mathrm{~Hz}$ (strong involuntary contraction) to increase muscle mass. ${ }^{19}$ The amplitude is dependent on the position of the feet on the vibration platform between 0 and $\pm 3.9 \mathrm{~mm}$ (peak-to-peak displacement is maximum $7.8 \mathrm{~mm}$ ). Peak acceleration related to frequency is $1.57 \mathrm{~g}$ for $10 \mathrm{~Hz}$ and $9.81 \mathrm{~g}$ for $25 \mathrm{~Hz}$. Children without standing ability use a vibrating platform combined with a tilt table. The tilting angle is individually adjustable according to the weight bearing ability ( 0 to 90 degrees).

During the first year of training, assessments are applied at $\mathrm{M} 0$, after 6 months of home training at M6, and at M12, in a 6month follow-up ( - Fig. 1). This has been previously described ${ }^{9}$ and will not be analyzed here. The treatment can be repeated after initial participation. The training during the consecutive years is shorter, since the child and the family are familiar with the program already and consists of an initial inpatient stay of only 1 week and a 4-month home-based WBV training (-Fig. 1). Assessments are applied at M0, after 4 months of home training at M4, and at M12, after an 8-month follow-up (- Fig. 1). Every repetition of the rehabilitation treatment is optional and can be initiated at any time after completion of the first year, when indicated by the physicians of the Centre of Prevention and Rehabilitation of the University Hospital of Cologne.

\section{Assessment of Motor Function by the Gross Motor Function Measure}

The Gross Motor Function Classification System (GMFCS) was used to classify the children according to their motor function level. The GMFCS consists of a 5-point ordinal scale, designated as I to V. ${ }^{20}$ Children with GMFCS levels I and II can walk without support or with limitations, respectively; children with levels III and IV can only walk using handheld mobility devices or powered mobility; children classified as level $\mathrm{V}$ are only passively transported in a manual wheelchair and show deficits in head and trunk control. ${ }^{20}$

Motor function was measured by the Gross Motor Function Measure (GMFM). The GMFM-66 is an observational clinical measure to evaluate gross motor function in children with CP. ${ }^{6}$ It consists of 66 motor tasks (items) and is validated and commonly used to quantify motor skills in children with $\mathrm{CP}$ (maximum score, 100 points). ${ }^{6,21,22}$ The results of the individual items are analyzed using the Gross Motor Ability Estimator (versions 1 and 2) Scoring Software for the GMFM (CanChild, McMaster University, Ontario, Canada). We only regarded measurements as valid, when at least 20 items (of 66) are examined and scored. Russell et al proposed that 13 completed items would be sufficient, but they emphasized that more items tested correlate with higher accuracy. ${ }^{23}$

An increase of the GMFM-66 score is generally interpreted as a response to intervention. However, it should be noted 
that children with CP typically show an increase in GMFM-66 scores up to the age of 7 to 10 years under standard of care. ${ }^{8,24,25}$ After the age of 7 to 10 years, most children with $\mathrm{CP}$ show a plateau in their motor development in the GMFM$66^{8,24,25}$ and children with GMFCS levels III, IV, and V might even decline. ${ }^{22}$ Therefore, to evaluate the real additional effect of an intervention in this population on motor function measured by the GMFM-66, the "expected development" should be considered. Otherwise, one would erroneously consider the increase in GMFM-66 score, which is expected in children anyway (up to the age 7 or 10 years), as a positive effect of the intervention. Therefore, for the analysis of motor development measured by the GMFM-66, over time, we used our proposed method ${ }^{9}$ that allowed the quantification of the progress of GMFM-66 scores to differentiate between possible treatment effects and expected development under standard of care. ${ }^{9}$ Using this method, the individual GMFM66 progression between two time points ( 6 months apart) can be quantified by a $Z$-score, taking into account the variability of individual development. This $Z$-score can then be evaluated according to the same criteria that Cohen proposed for the ES Cohen's d (see the Statistics section). ${ }^{26}$ The $Z$-score can therefore be regarded as equivalent to the value of the individual therapeutic ES (standardized response mean [SRM]); mathematical proof can be found in the supplement of Duran et al. $^{9}$

\section{Statistics}

In the first step of analysis, we used Cohen's d ES to quantify differences in the two groups (before M0 and after M4 intervention, or follow-up [standard of care] at M4-M12). The ES quantifies the size of GMFM-66 difference pre- and postintervention. ES is standardized, unit free, independent of sample size, and its value is near 0 , if the null hypothesis of the associated test was not rejected. ${ }^{26}$ ES is interpreted as follows:

- Trivial $(E S<0.20)$.

- Small (ES $\geq 0.20$ to $<0.50$ ).

- Medium (ES $\geq 0.50$ to $<0.80$ ).

- Large ( $E S \geq 0.80)$.

To separate responder from nonresponder, we defined nonresponder by $\mathrm{ES}<0.20$ and responder by $\mathrm{ES} \geq 0.20$ and calculated group specific ES for both years.

In the second step of analysis, we additionally applied the individual ES based on our proposed reference centiles described before, ${ }^{9}$ taking into account the expected development under standard of care for the individual child in a time period of 6 months. We used both methods to reassess validation with data of the second and third years and show accuracy of the results. An example for calculation is shown below. The individual ES is calculated by the following formula:

For dependent samples:

$$
\text { Individual ES }=\frac{\text { Mean change score }}{\text { standard deviation of change score }} \text {, }
$$

Where change score is the difference of the measures between two time points $t_{2}$ and $t_{1}$ :

$$
\text { Change score }=Z_{2}-Z_{1}
$$

\section{Example for Calculation of the Individual Effect Size According to Duran et al}

In the following, an example of a girl with bilateral spastic CP (GMFCS level I) is described to demonstrate the method. She participated in the first year of "Auf die Beine" (6 months of training instead of four months in repeated years). She started training with a GMFM-66 score of 85.2 at the age of 6 years and 8 months (M0) and improved to a score of 89.7 after 6 months of training (M6). Using the reference centiles proposed, ${ }^{9}$ the $Z$-scores for the GMFM-66 at M0 and M6 can be read off (-Fig. 2 ):

$$
\mathrm{Z}_{1}=2.10 \text { and } \mathrm{Z}_{2}=2.30
$$

The standard deviation (SD) of centile change can also be read off at the bottom dashed line in -Fig. 2 with 0.19 at the age of 6 years and 8 months in this case. According to Eq. (1), the Z-score of centile change (individual ES $)=(2.30-2.10) /$ $0.19=1.04$.

As described before, the $Z$-score can be regarded as equivalent to the value of the individual ES (SRM). ${ }^{9}$ According Cohen, ${ }^{26}$ the value of 1.04 correspondents to a large $(\geq 0.8)$ individual ES (see formula in Statistics section).

All calculations were performed with RStudio version 1.2.5001 in conjunction with $\mathrm{R}$ version 3.6.1 ( $\mathrm{R}$ Foundation for Statistical Computing, Vienna, Austria) and effsize (version 0.7.6). ${ }^{27-29}$ The results are presented as mean ( \pm SD) or count (relative frequency) or ES. Fisher's exact test was used in nominal factors; otherwise the Kruskal-Wallis test was used for calculating statistical significance. The KruskalWallis test (analysis of variance) tests whether an ordinally scaled variable originates from a common population. It is based on rank sums and can be used to compare more than two groups.

\section{Study Population}

For the first repetition (second year of training), at M0, 262 participants fulfilled the inclusion criteria. Of them, 115 $(43,89 \%)$ participants were female, the mean age was $7.2 \pm 2.2$ years, the mean height was $116.5 \pm 14.4 \mathrm{~cm}$, and the mean body mass index (BMI) was $15.3 \pm 2.5 \mathrm{~kg} / \mathrm{m}^{2}$ (-Table 1). Most participants were classified as bilateral spastic CP (71.37\%) followed by "nonclassifiable" (12.21\%), unilateral spastic CP (7.25\%), dyskinetic CP (4.96\%), and ataxic CP (4.20\%). The distribution of the GMFCS levels was as follows: GMFCS levels I (7.63\%), II (21.37\%), III (44.66\%), IV (24.05\%), and V (2.29\%).

For the second repetition (third year of training), at M0, 86 participants fulfilled the inclusion criteria. Of them, 37 $(43,02 \%)$ participants were female, the mean age was $8.4 \pm 2.4$ years, mean height was $122.5 \pm 14.2 \mathrm{~cm}$, and mean BMI was $16.2 \pm 3.4 \mathrm{~kg} / \mathrm{m}^{2}$ (-Table 2). Most participants were classified as bilateral spastic $\mathrm{CP}(76.74 \%)$ followed by unilateral spastic (9.30\%) and "nonclassifiable" CP (9.30\%), ataxic (2.33\%), and dyskinetic CP (2.33\%). The distribution of 


\section{GMFM-66 in children with CP}

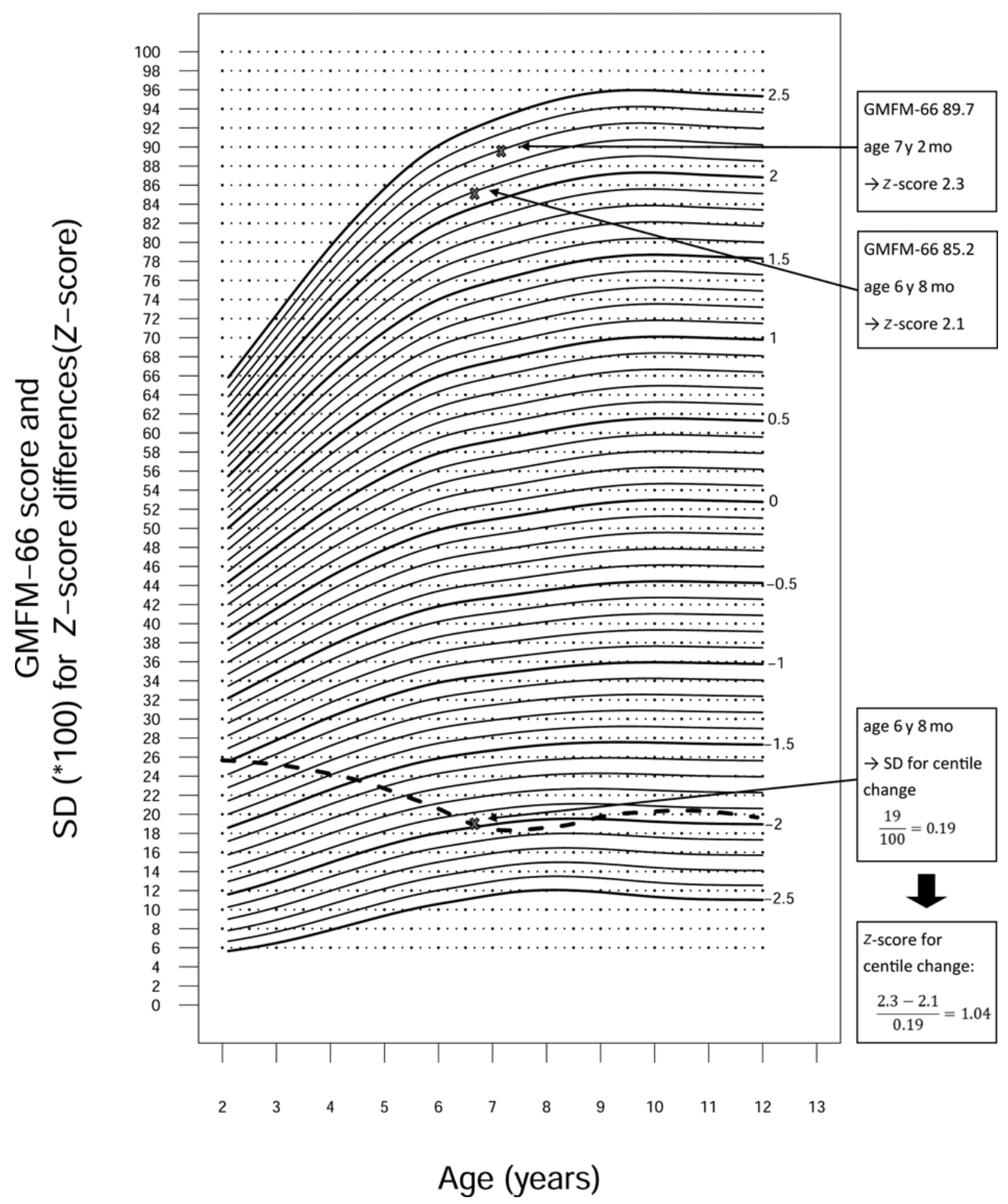

Fig. 2 The figure illustrates the usage of the reference centiles of Duren et al. ${ }^{9}$ First, the two (M0 and M6) GMFM-66 scores are entered (two upper crosses: age $1[\mathrm{M} 0]=6$ years 8 months, age $2[\mathrm{M} 6]=7$ years 2 months). The corresponding $Z$-scores can be read off at the left $\left(Z_{1}=2.1\right.$ and $Z_{2}=2.3$ ). The age-dependent standard deviation (SD) of the centile change can be read off the dashed curve at the bottom at the age of the child at the first GMFM-66 measurement ( $S D=0.19)$. Then, with the formula $Z_{2}-Z_{1} / S D$, the $Z$-score for the centile change (individual $\left.E S\right)$ can be calculated (=1.04). CP, cerebral palsy; GMFM, gross motor function measure. 
412 Effect of Long-Term Repeated Interval Rehabilitation on the GMFM-66 Stark et al.

Table 1 Study population and the effect of the second year of the rehabilitation program on GMFM-66 in children with CP

\begin{tabular}{|c|c|c|c|c|c|c|}
\hline \multirow[t]{2}{*}{ Factor $^{\mathrm{a}}$} & \multirow[t]{2}{*}{ All } & \multicolumn{5}{|c|}{ Grouped by individual effect size at M4 } \\
\hline & & $E S<0.2$ & $0.2 \leq \mathrm{ES}<0.5$ & $0.5 \leq \mathrm{ES}<0.8$ & $\mathrm{ES} \geq 0.8$ & $p^{\mathrm{b}}$ \\
\hline$n$ & 262 & 125 & 52 & 32 & 53 & NA \\
\hline Female & 115 & 59 & 17 & 13 & 26 & 0.290 \\
\hline Age $(y)$ & $7.2(2.2)$ & $7.0(2.1)$ & $7.2(2.4)$ & $7.0(1.8)$ & $7.6(2.4)$ & 0.465 \\
\hline Height $(\mathrm{cm})$ & $116.5(14.4)$ & $115.1(14.1)$ & 117.5 (13.7) & $16.6(12.0)$ & $118.7(17.1)$ & 0.467 \\
\hline BMI $\left(\mathrm{kg} / \mathrm{m}^{2}\right)$ & $15.3(2.5)$ & $15.1(2.6)$ & $15.4(2.4)$ & $15.1(2.3)$ & $15.7(2.8)$ & 0.541 \\
\hline \multicolumn{7}{|l|}{ CP subtype } \\
\hline Bilateral & 187 & 91 & 34 & 26 & 36 & 0.548 \\
\hline Unilateral & 19 & 11 & 4 & 0 & 4 & \\
\hline Dyskinetic & 13 & 6 & 3 & 0 & 4 & \\
\hline Ataxic & 11 & 3 & 2 & 2 & 4 & \\
\hline Nonclassifiable & 32 & 14 & 9 & 4 & 5 & \\
\hline \multicolumn{7}{|l|}{ GMFCS levels } \\
\hline I & 20 & 6 & 2 & 2 & 10 & 0.175 \\
\hline II & 56 & 22 & 15 & 7 & 12 & \\
\hline III & 117 & 60 & 24 & 14 & 19 & \\
\hline IV & 63 & 32 & 11 & 9 & 11 & \\
\hline $\mathrm{V}$ & 6 & 5 & 0 & 0 & 1 & \\
\hline
\end{tabular}

Abbreviations: BMI, body mass index; CP, cerebral palsy; ES, effect size; GMFM-66, gross motor function measure-66 items; GMFCS, gross motor function classification system; M4, 4 months after initiation of treatment; NA, not applicable; SD, standard deviation.

Note: Data are presented as mean ( \pm SD) and count (frequency).

${ }^{a}$ The factors were determined at M0 (baseline of treatment), see methods for explanation of M0.

bFisher's exact test was used in nominal factors, otherwise Kruskal-Wallis test was used.

the GMFCS levels was as follows: GMFCS levels I (3.49\%), II (23.26\%), III (44.19\%), IV (26.74\%), and V (2.33\%).

The characteristics of the two patient groups (completed second and third years of training) did not differ significantly regarding GMFCS levels and type of $\mathrm{CP}$ (-Tables 1 and $\mathbf{2}$ ).

\section{Results}

\section{Second Year (M4)}

After the treatment phase at M4 in the second year $(n=262)$, according to Eq. (1), the Cohen's d ES was 0.448 (95\% confidence interval [CI]: 0.622-0.274) for group difference, revealing a small (almost medium) effect. Using the individual ES with our proposed method, the ES was 0.336 (95\% CI: 0.246-0.426), meaning a small effect. Z-scores for the GMFM-66 between M0 and M4 improved significantly $(p<0.0001)$ with a mean difference of 0.069 (95\% CI: 0.087-0.050).

\section{Second Year Follow-up (M12)}

After the follow-up phase (standard of care) at M12 in the second year, according to Eq. (1), the Cohen's d ES was 0.071 (95\% CI: 0.100-0.243) for group difference, meaning a negligible effect. Using the individual ES with our proposed method, the ES was 0.087 (95\% CI: 0.054-0.227), meaning a negligible effect. Accordingly, Z-scores for the GMFM-66 between M4 and M12 remained stable with mean 0.236 (95\% CI: $0.128-0.343$ ) to mean 0.251 (95\% CI: 0.140-0.362) with no significant change $(p=0.250)$ and a mean difference of -0.016 ( $95 \% \mathrm{CI}:-0.042$ to 0.011 ).

\section{Third Year (M4)}

After the treatment phase at M4 in the third year $(n=86)$, according to Eq. (1), the Cohen's d ES was 0.513 (95\% CI: 0.819-0.207) for group difference, meaning a medium effect. Using the individual ES with our proposed method for the third year, the ES was 0.379 (95\% CI: 0.221-0.536), revealing a small effect. $Z$-scores for the GMFM-66 between M0 and M4 improved significantly $(p<0.0001)$ with a mean difference of 0.075 (95\% CI: 0.044-0.107).

\section{Third Year Follow-up (M12)}

After the follow-up phase (standard of care) at M12 in the third year, according to Eq. (1), Cohen's d ES was 0.038 (95\% CI: 0.263-0.339) for group difference, meaning a negligible effect. Using the individual ES with our proposed method, the ES was 0.025 (95\% CI: 0.127-0.179), showing a negligible effect. Accordingly, Z-scores for the GMFM-66 between M4 and M12 remained stable with mean 0.169 (95\% CI: $0.001-$ 0.340 ) to mean 0.174 (95\% CI: 0.006-0.346) with no significant change $(p=0.725)$ with a mean difference of -0.005 (95\% CI: -0.03 to -0.025 ). 
Table 2 Study population and the effect of the third year of the rehabilitation program on GMFM-66 in children with CP

\begin{tabular}{|c|c|c|c|c|c|c|}
\hline \multirow[t]{2}{*}{ Factor $^{\mathrm{a}}$} & \multirow[t]{2}{*}{ All } & \multicolumn{5}{|c|}{ Grouped by individual effect size (ES) at M4 } \\
\hline & & $E S<0.2$ & $0.2 \leq \mathrm{ES}<0.5$ & $0.5 \leq \mathrm{ES}<0.8$ & $E S \geq 0.8$ & $p^{\mathrm{b}}$ \\
\hline$n$ & 86 & 43 & 17 & 6 & 20 & NA \\
\hline Female & 37 & 19 & 7 & 3 & 8 & 0.986 \\
\hline Age (y) & $8.4(2.4)$ & $7.9(2.3)$ & $9.1(2.4)$ & $10.3(2.0)$ & $8.3(2.4)$ & 0.068 \\
\hline Height (cm) & $122.5(14.2)$ & $119.1(12.8)$ & $127.4(15.2)$ & $132.8(15.8)$ & $122.6(14.0)$ & 0.080 \\
\hline BMI $\left(\mathrm{kg} / \mathrm{m}^{2}\right)$ & $16.2(3.4)$ & $15.7(3.7)$ & $17.5(3.4)$ & $15.5(3.4)$ & $16.5(2.8)$ & 0.165 \\
\hline CP subtype & & & & & & 0.357 \\
\hline Bilateral & 66 & 36 & 11 & 4 & 15 & \\
\hline Unilateral & 8 & 3 & 2 & 0 & 3 & \\
\hline Dyskinetic & 2 & 1 & 1 & 0 & 0 & \\
\hline Ataxic & 2 & 0 & 1 & 0 & 1 & \\
\hline Nonclassifiable & 8 & 3 & 2 & 2 & 1 & \\
\hline GMFCS level & & & & & & 0.062 \\
\hline I & 3 & 0 & 1 & 0 & 2 & \\
\hline II & 20 & 7 & 4 & 1 & 8 & \\
\hline III & 38 & 21 & 7 & 2 & 8 & \\
\hline IV & 23 & 15 & 4 & 2 & 2 & \\
\hline V & 2 & 0 & 1 & 1 & 0 & \\
\hline
\end{tabular}

Abbreviations: BMI, body mass index; CP, cerebral palsy; ES, effect size; GMFM-66, gross motor function measure-66 items; GMFCS, gross motor function classification system; M4, 4 months after initiation of treatment; NA, not applicable; SD, standard deviation.

Data are presented as mean $( \pm S D)$ and count.

${ }^{\mathrm{a}}$ The factors were determined at M0 (baseline of treatment), see methods for explanation of M0.

bFisher's exact test was used in nominal factors, otherwise Kruskal-Wallis test was used.

\section{Responder/Nonresponder}

The demographic characteristics between the children with no, moderate, or large ES at M4 did not differ significantly at M0 (-Tables 1 and 2). Less-affected children (GMFCS levels I and II) tend to benefit more than children with GMFCS levels III to $\mathrm{V}$ but not significantly.

In the second year of rehabilitation, 125 children were responder, defined by $E S \geq 0.20$, and 137 children were nonresponder, defined by $\mathrm{ES}<0.20$. Mean individual ES of the group of nonresponder was -0.212 (95\% CI: -0.281 to -0.142 ) and for responder 0.836 (95\% CI: 0.731-0.941).

In the third year of rehabilitation, 43 children were responder, defined by ES $\geq 0.20$, and 43 children were nonresponder, defined by ES $<0.20$. Mean individual ES of the group of nonresponder was -0.124 (95\% CI: -0.185 to 0.063 ) and for responder 0.881 (95\% CI: 0.656-1.106).

\section{Characteristics of Children Repeating Rehabilitation}

As described above, the overall mean individual ES for the whole group of the second year of rehabilitation was 0.336 (95\% CI: 0.246-0.426) at M4. For the group of 86 children only, which repeated rehabilitation for the third year, the ES for the second year was 0.348 (95\% CI: 0.192-0.505) at M4. There is no correlation of the individual ES between the first (M0M6) and the second year of rehabilitation (M0-M4, $r=0.04$, $p=0.458$ ), or between the second (M0-M4) and the third year of rehabilitation (M0-M4, $r=0.18, p=0.092$; - Fig. 3 ).

\section{Discussion}

In this observational analysis, we quantified the changes of GMFM-66 scores of a long-term repeated interval rehabilitation program with home-based, vibration-assisted training, "Auf die Beine" in children with CP. We could demonstrate a small (almost medium) effect after the active training period at M4 for the second year and again a medium effect after the active training period (M4) of the third year. The effect of this rehabilitation program on the GMFM-66 in children with $\mathrm{CP}$ has previously shown an almost large ES for the first year of rehabilitation after 6 months of intensive training. ${ }^{9}$

A very important observation was that no additional treatment effect could be observed in the follow-up period (standard of care) of 8 months (M12) in both years. Patients stayed stable on their newly achieved motor level after intervention and further followed their "standard-ofcare"-predicted development. This means that the rehabilitation program "Auf die Beine" has an additional positive effect on motor development measured by the GMFM-66 (on top of the expected natural development under standard of care). When the program ends, the patients follow their predicted development under standard of care without any additional treatment effect anymore.

In further analysis, using the individual ES, we could show that $50 \%$ of the children in both groups (second and third 

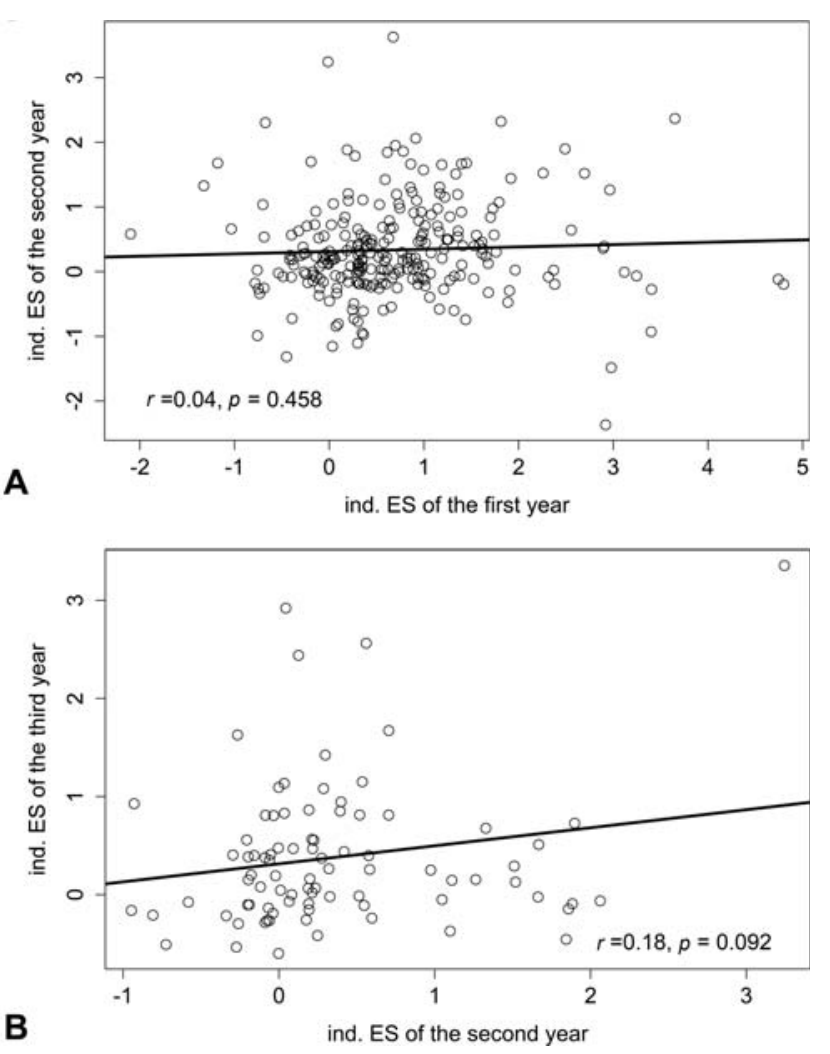

Fig. 3 Correlation of the individual (ind.) effect size (ES) of the first and second year (A) and the second and third year (B) of the rehabilitation program.

years) were responder and showed large ES ( $>0.8)$. Meaning that $50 \%$ showed a large additional effect of the rehabilitation program on top of the expected development under standard of care. This does not mean that the other half did not show any progression in the period; it just shows that the other half, which we defined as nonresponder by an ES $>0.2$ (trivial effect), followed their expected natural development under standard of care (including all available treatment options in Germany).

In both analyses (previous analysis of first year of rehabilitation $^{9}$ and this analysis), we could show comparable results for using Cohen's d ES and the individual ES based on the reference centiles proposed ${ }^{9}$; which shows that our proposed method is valid. Due to the small sample size for the second and third years, we were not able to create reference percentiles for the 4-month period. Therefore, we decided to utilize the published centiles based on the 6-month interval. ${ }^{9}$ Thus, SD values might be overestimated, since less-spontaneous variation is expected in a period of 4 months rather than in a 6month period. In this case, the individual ES revealed in our study findings could be underestimated which is confirmed by the higher Cohen's d ES, for example, we found a Cohen's d ES for the second year of training of 0.448 (small/almost medium) and using the individual ES with our proposed method the ES was 0.336 , meaning a small effect.

The significantly improved motor development of patients receiving the rehabilitation treatment "Auf die Beine" is likely to be due to the intervention. The GMFM66 reference percentiles ${ }^{9}$ predict motor development under standard of care. After improved motor performance after intensive treatment, the children follow their predicted motor development under standard of care on a higher motor level as described above. However, a treatment effect of the first year measured by the GMFM-66 was no predictor for an effect of the second year. The same was true for the second and third years. Meaning the decision on repeating the rehabilitation program is not based on the GMFM-66 alone, but on many other factors, like other assessments (minute walking tests, gait analysis, other objective, and subjective measures) and individual factors. Also, the ES for the GMFM66 for the entire group of the second year repeaters compared with the third year repeaters alone in the second year shows no difference.

Overall the repeated rehabilitation treatment for a second and third years shows smaller effect than the results of the first year. ${ }^{9}$ It is unclear whether repeated interventions show smaller effects due to the exhausted treatment potential or the underestimation is due to the missing reference centiles for the training period of 4 months. This should be investigated in future studies. Additionally, children in the second and third years are older because it is the same cohort chronologically followed. It is known that motor development potential shows a plateau with increasing age. ${ }^{22}$

No other therapeutic program, neither conservative nor pharmacological nor surgical, could present the treatment results in children and adolescents with $\mathrm{CP}$ taking into account the expected development under standard of care. In addition, there are very few long-term studies in the literature regarding $\mathrm{CP}$ treatment. ${ }^{30-34}$ Even current protocols, such as proposed by García-Galant et al fail to include a proper long-term assessment or follow-up. ${ }^{35}$ Even regarding the use of invasive treatments, such as intrathecal Baclofen's or selective dorsal rhizotomy (SDR), that should actually had been better followed-up, the evidence is very scarce and insufficient. ${ }^{36}$ Therefore, we are not able to compare our data to other results. Due to the high socioeconomic importance of this disease for the health system, however, it is highly relevant to review the effectiveness of the therapeutic programs. This should be investigated in future studies.

\section{Study Limitations}

This study is limited by the description of a multimodular physiotherapy program. It is not possible to assess which intervention was the most efficient. The patients were a heterogeneous group with different levels of mobility and lack of a control group. However, the intention was to show systematic evaluation of clinical care for best-possible treatment options for children with motor disorders like CP. Because it is a voluntary decision by the families to participate for repeated years, the sample is highly motivated and not generalizable to the general population.

\section{Conclusion}

In this observational analysis, we quantified changes of GMFM-66 scores of a long-term repeated interval 
rehabilitation program with home-based, vibration-assisted training "Auf die Beine" in children with CP. We could demonstrate that $50 \%$ were responder and showed large ES $(>0.8)$. The results of this observational study can be used to generate hypothesis of the effectiveness of the treatment. However, randomized controlled studies are needed to prove the generated hypothesis.

\section{Author Contributions}

All authors fulfil the four criteria for authorship recommended by ICJME and all who meet the four criteria are identified as authors.

\section{Ethical Approval}

This study was approved by the Ethics Committee of the University of Cologne, Germany.

\section{Funding}

This research received no specific grant from any funding agency in the public, commercial, or not-for-profit sectors.

\section{Conflicts of Interest}

E.S. is the medical director of the UniReha GmbH Centre of Prevention and Rehabilitation (University of Cologne) and UniReha GmbH Centre of Prevention and Rehabilitation (University of Cologne) employ I.D., K.S., and C.S.. Novotec Medical $\mathrm{GmbH}$ is supporting research at UniReha $\mathrm{GmbH}$. C. $\mathrm{S}$. reports to have received travel support from Novotec Medical $\mathrm{GmbH}$ to present research at medical conferences. She received research grants by Novotec Medical GmbH. She has no further financial relationships with Novotec Medical GmbH. No other disclosures are reported.

\section{Acknowledgments}

We would like to thank the physiotherapists of the UniReha $\mathrm{GmbH}$, Centre of Prevention and Rehabilitation, program "Auf die Beine" (University of Cologne) for their high dedication to their work and assessment of the GMFM66. We thank Valérie Adames for GMFM-66 quality management. We thank Angelika Stabrey for statistical assistance and data handling and Ida Alperstedt for data input. We thank all patients and families for participating in the Cologne physiotherapy treatment program "Auf die Beine."

\section{References}

1 Krägeloh-Mann I, Cans C. Cerebral palsy update. Brain Dev 2009; 31(07):537-544

2 Centers for Disease Control and Prevention (CDC). Economic costs associated with mental retardation, cerebral palsy, hearing loss, and vision impairment-United States, 2003. MMWR Morb Mortal Wkly Rep 2004;53(03):57-59

3 Alotaibi M, Long T, Kennedy E, Bavishi S. The efficacy of GMFM-88 and GMFM-66 to detect changes in gross motor function in children with cerebral palsy (CP): a literature review. Disabil Rehabil 2014;36(08):617-627

4 Ryan JM, Cassidy EE, Noorduyn SG, O'Connell NE. Exercise interventions for cerebral palsy. Cochrane Database Syst Rev 2017;6: CD011660
5 Maltais DB, Wiart L, Fowler E, Verschuren O, Damiano DL. Healthrelated physical fitness for children with cerebral palsy. J Child Neurol 2014;29(08):1091-1100

6 Russell DJ, Rosenbaum PL, Avery LM, Lane M. Gross Motor Function Measure (GMFM-66 \& GMFM-88) User's Manual. London, United Kingdom: Mac Keith Press; 2002

7 Palisano RJ, Hanna SE, Rosenbaum PL, et al. Validation of a model of gross motor function for children with cerebral palsy. Phys Ther 2000;80(10):974-985

8 Rosenbaum PL, Walter SD, Hanna SE, et al. Prognosis for gross motor function in cerebral palsy: creation of motor development curves. JAMA 2002;288(11):1357-1363

9 Duran I, Stark C, Martakis K, Hamacher S, Semler O, Schoenau E. Reference centiles for the gross motor function measure and identification of therapeutic effects in children with cerebral palsy. J Eval Clin Pract 2019;25(01):78-87

10 Stark C, Hoyer-Kuhn HK, Semler O, et al. Neuromuscular training based on whole body vibration in children with spina bifida: a retrospective analysis of a new physiotherapy treatment program. Childs Nerv Syst 2015;31(02):301-309

11 Stark C, Nikopoulou-Smyrni P, Stabrey A, Semler O, Schoenau E. Effect of a new physiotherapy concept on bone mineral density, muscle force and gross motor function in children with bilateral cerebral palsy. J Musculoskelet Neuronal Interact 2010;10(02): 151-158

12 Stark C, Semler O, Duran I, et al. Intervallrehabilitation mit häuslichem Training bei Kindern mit Zerebralparese. Monatsschr Kinderheilkd 2013;161:625-632

13 Hoyer-Kuhn H, Semler O, Stark C, Struebing N, Goebel O, Schoenau E. A specialized rehabilitation approach improves mobility in children with osteogenesis imperfecta. J Musculoskelet Neuronal Interact 2014;14(04):445-453

14 Stark C, Duran I, Cirak S, et al. Vibration-assisted home training program for children with spinal muscular atrophy. Child Neurol Open 2018;5:X18780477

15 Ritzmann R, Kramer A, Gollhofer A, Taube W. The effect of whole body vibration on the H-reflex, the stretch reflex, and the shortlatency response during hopping. Scand J Med Sci Sports 2013;23 (03):331-339

16 Ritzmann R, Gollhofer A, Kramer A. The influence of vibration type, frequency, body position and additional load on the neuromuscular activity during whole body vibration. Eur J Appl Physiol 2013;113(01):1-11

17 Krause A, Schönau E, Gollhofer A, et al. Alleviation of motor impairments in patients with cerebral palsy: acute effects of whole-body vibration on stretch reflex response, voluntary muscle activation and mobility. Front Neurol 2017;8:416

18 Krause A, Gollhofer A, Freyler K, Jablonka L, Ritzmann R. Acute corticospinal and spinal modulation after whole body vibration. J Musculoskelet Neuronal Interact 2016;16(04):327-338

19 Stark C, Duran I, Schoenau E. Pediatric rehabilitation. In: Rittweger J, ed. Manual of Vibration Exercise and Vibration Therapy. 1st ed. Cologne, Germany: Springer Nature; 2020:285-318

20 Rosenbaum PL, Palisano RJ, Bartlett DJ, Galuppi BE, Russell DJ. Development of the gross motor function classification system for cerebral palsy. Dev Med Child Neurol 2008;50(04):249-253

21 Russell DJ, Avery LM, Rosenbaum PL, Raina PS, Walter SD, Palisano RJ. Improved scaling of the gross motor function measure for children with cerebral palsy: evidence of reliability and validity. Phys Ther 2000;80(09):873-885

22 Hanna SE, Rosenbaum PL, Bartlett DJ, et al. Stability and decline in gross motor function among children and youth with cerebral palsy aged 2 to 21 years. Dev Med Child Neurol 2009;51(04): 295-302

23 Russell DJ, Avery LM, Walter SD, et al. Development and validation of item sets to improve efficiency of administration of the 66-item Gross Motor Function Measure in children with cerebral palsy. Dev Med Child Neurol 2010;52(02):e48-e54 
24 Hanna SE, Bartlett DJ, Rivard LM, Russell DJ. Reference curves for the Gross Motor Function Measure: percentiles for clinical description and tracking over time among children with cerebral palsy. Phys Ther 2008;88(05):596-607

25 Smits DW, Gorter JW, Hanna SE, et al; PERRIN Plus Study Group. Longitudinal development of gross motor function among Dutch children and young adults with cerebral palsy: an investigation of motor growth curves. Dev Med Child Neurol 2013;55(04):378-384

26 Cohen J. Statistical Power Analysis for the Behavioral Sciences. 2nd ed. Hillsdale, NJ: Erlbaum; 1988

27 Rigby RA, Stasinopoulos DM. Generalized additive models for location, scale and shape. Appl Stat 2005;54(03):507-554

28 Robin X, Turck N, Hainard A, et al. pROC: an open-source package for $\mathrm{R}$ and $\mathrm{S}+$ to analyze and compare ROC curves. BMC Bioinformatics 2011;12:77-2105

29 Torchiano M. Effsize: Efficient Effect Size Computation. Available at: https://zenodo.cern.ch/record/196082. Accessed July 16 , 2020

30 Liu XC, Embrey D, Tassone C, et al. Long-term effects of orthoses use on the changes of foot and ankle joint motions of children with spastic cerebral palsy. PM R 2018;10(03):269-275

31 Sung KH, Kwon SS, Chung CY, Lee KM, Cho GH, Park MS. Long-term outcomes over 10 years after femoral derotation osteotomy in ambulatory children with cerebral palsy. Gait Posture 2018;64 (May):119-125

32 Eliasson AC, Holmefur M. The influence of early modified constraint-induced movement therapy training on the longitudinal development of hand function in children with unilateral cerebral palsy. Dev Med Child Neurol 2015;57(01):89-94

33 Fattal-Valevski A, Domenievitz D, Giladi N, Wientroub S, Hayek S. Long-term effect of repeated injections of botulinum toxin in children with cerebral palsy: a prospective study. J Child Orthop 2008;2(01):29-35

34 Bolster EA, van Schie PE, Becher JG, van Ouwerkerk WJ, Strijers RL, Vermeulen RJ. Long-term effect of selective dorsal rhizotomy on gross motor function in ambulant children with spastic bilateral cerebral palsy, compared with reference centiles. Dev Med Child Neurol 2013;55(07):610-616

35 García-Galant M, Blasco M, Reid L, et al. Study protocol of a randomized controlled trial of home-based computerized executive function training for children with cerebral palsy. BMC Pediatr 2020;20(01):9

36 Davidson B, Schoen N, Sedighim S, et al. Intrathecal baclofen versus selective dorsal rhizotomy for children with cerebral palsy who are nonambulant: a systematic review. J Neurosurg Pediatr 2019;25(01):1-9 